\title{
Complications of tube thoracostomy in trauma
}

\author{
R C Bailey
}

\begin{abstract}
Objective-To assess the complication rate of tube thoracostomy in trauma. To consider whether this rate is high enough to support a selective reduction in the indications for tube thoracostomy in trauma.

Methods-A retrospective case series of all trauma patients who underwent tube thoracostomy during a 12 month period at a large UK teaching hospital with an accident and emergency (A\&E) department seeing in excess of 125000 new patients/ year. These patients were identified using the hospital audit department computerised retrieval system supplemented by a hand search of both the data collected for the Major Trauma Outcome Study and the A\&E admission unit log book. The notes were assessed with regard to the incidence of complications, which were divided into insertional, infective, and positional.

Results-Fifty seven chest drains were placed in 47 patients over the 12 month period. Seven patients who died within 48 hours of drain insertion were excluded. The commonest indications for tube thoracostomy were pneumothorax $(54 \%)$ and haemothorax $(20 \%) ; 90 \%$ of tubes were placed as a result of blunt trauma. The overall complication rate of the procedure was $30 \%$. There were no insertional complications and only one (2\%) major complication, which was empyema thoracis. Conclusion-This study reveals no persuasive evidence to support a selective reduction in the indications for tube thoracostomy in trauma. A larger study to confirm or refute these findings must be performed before any change in established safe practice.

(F Accid Emerg Med 2000;17:111-114)
\end{abstract}

Keywords: tube thoracostomy; trauma; complications

Fewer than $10 \%$ of blunt chest injuries and $15 \%-30 \%$ of penetrating chest injuries require thoracotomy. ${ }^{1}$ Initially, the majority of these injuries can be effectively managed by careful assessment of the airway, breathing, and circulation making appropriate interventions as indicated according to Advanced Trauma Life Support (ATLS) principles. The insertion of an appropriately sized chest drain has played a pivotal role in this process. Recently, the necessity of chest drain insertion for a proportion of traumatic pneumothoraces has been challenged. ${ }^{2}$ The justification for this proposed change is the high complication rate some authors associate with tube thoracostomy. ${ }^{3-6}$
These complications can be categorised as insertional, positional, or infective. ${ }^{7}$ The insertion of a chest drain in trauma is a relatively common intervention performed in the accident and emergency (A\&E) department of Queens Medical Centre, Nottingham. In view of the above suggestion it was therefore appropriate to assess the complication rate of tube thoracostomy in this institution.

\section{Methods}

A retrospective case series of all trauma patients who underwent tube thoracostomy for any indication between 1 January 1996 and 31 December 1996 was conducted. These patients were identified by the hospital audit department's computerised retrieval system by sorting for the tube thoracostomy procedure code (the audit department retrospectively scrutinise the documentation of all trauma admissions and code them for procedures performed during their admission). This was cross referenced by both a hand search of the data collected for the Major Trauma Outcome Study and the A\&E unit log book. The latter records the details of all patients admitted with chest injuries not requiring intensive care or transfer to the thoracic surgery unit and thus identifies patients admitted with simple pneumothoraces who were discharged within three days.

The notes were reviewed for age, sex, mechanism of injury, site within the hospital where the procedure was performed, seniority of the operator, indication, duration of insertion, complications, and length of follow up. Indications included pneumothorax, haemothorax, and flail segment (table 1). Seven patients who died as a result of their injuries were excluded from analysis as they could not be evaluated for late complications. All seven patients underwent postmortem examination which confirmed the thoracostomy tubes were correctly sited and that no death was related to tube thoracostomy. Before their death, the hospital notes of these patients recorded no complications related to tube thoracostomy.

Complications were defined as insertional (for example, lung or other organ laceration or perforation, haemorrhage), positional (for example, extrathoracic placement, persistent haemothoraces or pneumothoraces), or infective (for example, minor wound infection,

Table 1 Indications for tube thoracostomy

\begin{tabular}{lc}
\hline & No (\%) \\
\hline Pneumothorax & $27(54)$ \\
Haemothorax & $10(20)$ \\
Flail segment & $9(18)$ \\
Tension pneumothorax & $3(6)$ \\
Multiple rib fractures & $1(2)$ \\
\hline
\end{tabular}


Table 2 Complications of tube thoracostomy

\begin{tabular}{lc}
\hline Insertional & 0 \\
Positional & \\
Post-removal pneumothorax & 3 \\
Drain dislodgement & 2 \\
Non-functional & \\
$\quad$ (I) Kinked & 1 \\
$\quad$ (II) Clotted & 1 \\
Collapse right lower lobe & 1 \\
Total & 8 \\
Infective & \\
Minor: drain site infection & 6 \\
Major: empyema thoracis & 1 \\
Total & 7 \\
Overall total & 15 \\
\hline
\end{tabular}

empyema thoracis). All chest drains were placed using the open technique recommended by ATLS and other expert authorities. ${ }^{189}$ Prophylactic antibiotics were not administered.

\section{Results}

A total of 57 chest drains were placed in 47 patients over the 12 month period. Seven patients who died within 48 hours of tube thoracostomy were excluded. Therefore the study evaluated 50 chest drains in 40 patients. Thirty patients had one chest drain and 10 patients had two.

Thirty nine thoracostomies were performed in the $A \& E$ department resuscitation room, and 11 on the intensive care unit or $A \& E$ ward. Table 1 lists the indications for tube thoracostomy. The commonest indications were pneumothorax (54\%) and haemothorax (20\%).

The mean age of the patients was 43 years and $74 \%$ were male. The duration of drain placement ranged from two to nine days with a mean of four days. Five $(10 \%)$ of the drains placed for simple pneumothoraces required the application of low pressure, high volume suction to facilitate full resolution of the pneumothorax. Ninety per cent were placed as a result of blunt trauma, $6 \%$ as a result of penetrating trauma, and $4 \%$ as a result of barotrauma. Sixty four per cent of procedures were performed by "senior" A\&E doctors (consultant, senior registrar, registrar, staff grade) and $20 \%$ by senior house officers. In $16 \%$ the grade of doctor was not known.

Twenty eight patients with isolated chest injuries had a mean admission duration of 8 days (range 3-35 days), and a mean follow up period of 7.5 weeks (range 1-56 weeks). Twelve patients whose chest injury was part of multiple trauma had a mean admission duration of 33 days (range 13-48 days), and a mean follow up period of 27 weeks (range 7-60 weeks). All patients were followed up until discharge from clinic with none defaulting.

The overall complication rate related to tube thoracostomy was $30 \%$. Table 2 lists the individual complications with their absolute numbers.

\section{Discussion}

A 30\% complication rate for tube thoracostomy seems unacceptably high, however, the type of complication encountered merits further analysis. Millikan et al retrospectively ana- lysed the notes of 447 patients who underwent tube thoracostomy between 1974 and $1978 .^{10}$ There were four $(1 \%)$ documented technical complications in the 447 patients. These consisted of two isolated diaphragmatic perforations, one lung laceration, and one patient whose misplaced drain perforated the diaphragm, avulsed a portion of the lesser curve of the stomach, and lacerated the liver. They also found 11 cases $(2.7 \%)$ of empyema. No patient died. Compared with the series of Millikan et $a l$, this series contained no insertional or "technical" complications and only one $(2 \%)$ case of empyema. This patient underwent a thoracotomy and decortication of the right lower lobe; there was no residual respiratory deficit at outpatient discharge 56 weeks later.

Since Hewett in 1876 first described continuous drainage of the chest via an intercostal tube, ${ }^{11}$ numerous techniques of insertion and complications, sometimes fatal, have been described. ${ }^{12-16}$ It is now generally accepted that the trocar has no part to play in the safe insertion of a chest drain, the preferred method being blunt and open. ${ }^{189}$ By employing this technique, particularly the correct site of insertion, blunt dissection above the rib, and the finger sweep to ensure the lung is not adherent to the chest wall, insertional complications should be eliminated. There have been no significant studies performed in this area since the widespread adoption of the blunt technique, and the absence of insertional complications in this series is therefore of note. "Senior" A\&E staff with ATLS training sited the majority (64\%) of drains in this series; this may have contributed to the absence of insertional complications.

Millikan et al did not describe "positional" complications in his series, Etoch et al encountered an overall complication rate of $16 \%$ per tube thoracostomy in their series in $1995 .^{3}$ However, only one case $(0.2 \%)$ had an insertional complication (lung laceration which required thoracotomy). A further seven cases $(1.5 \%)$ developed empyema.

Collop et al encountered 14 complications in 126 tube thoracostomies (11\% complication rate). ${ }^{7}$ Only one complication was insertional (lung laceration). Chan et al encountered 64 complications in 352 tube thoracostomies ( $18.2 \%$ complication rate), but no insertional complications. ${ }^{6}$

The incidence of thoracic empyema after tube thoracostomy reported in the literature varies widely, from $1 \%$ to $25 \% .{ }^{5}$ A rate of $2 \%$ encountered in this series seems consistent with other recent series $\left(1.1 \%^{6}, 2 \%{ }^{33}\right.$ and $\left.2.4 \%{ }^{10}\right)$. Nichols et al evaluated the safety and efficacy of antibiotics in reducing the infectious complication rate after tube thoracostomy for isolated chest trauma. ${ }^{17}$ They concluded those patients receiving prophylactic antibiotics had a significantly reduced infection rate compared with those given placebo. No significant adverse effects were seen in either group. Grover et al noted a $2.6 \%$ rate of empyema in patients randomised to receive prophylactic antibiotics after tube thoracostomy for penetrating chest trauma and a slightly higher rate 
in those receiving placebo. ${ }^{18}$ In his metaanalysis Fallon suggested that prophylactic antibiotics reduced the incidence of thoracic empyema after tube thoracostomy. ${ }^{19}$ However, Etoch et al detected no discernible difference in infection rates dependent on early antibiotic use. ${ }^{3}$ Similarly, in view of the infrequent incidence of empyema, they were unable to predict any particular factors that predisposed to infectious complications.

Closer analysis of the "positional" complications encountered in this series reveals three cases of recurrent pneumothorax after removal of the drain. Repeated tube thoracostomy without further complication successfully treated all three. One patient developed collapse of the right lower lobe of the lung that was successfully treated with physiotherapy resulting in full radiological and clinical recovery. Two drains were dislodged by patients and treated by repeated tube thoracostomy without further complication. Two of the drains became non-functional secondary to kinking and clotting and were replaced without further complication.

Of the seven infective complications, six were drain site infections (two diagnosed on clinical criteria, four with positive wound cultures: all Staphylococcus aureus), that responded to a course of oral flucloxacillin. The only complication to result in considerable morbidity was the thoracic empyema, which was treated successfully at thoracotomy with no residual respiratory deficit at discharge from follow up.

Etoch et al refer to the "liberal" definition of complications used in their series that displayed a $21 \%$ complication rate. $^{3}$ The $30 \%$ complication rate described here would be dramatically reduced by a less liberal definition of complications. If only complications resulting in significant morbidity were included it would fall to $2 \%$. Although it is possible that some complications may have developed after discharge and follow up, the mean follow up period of 7.5 weeks for isolated chest injuries and 27 weeks for those involved in multiple trauma makes this unlikely.

An area not covered by the ATLS manual is chest drain removal. ${ }^{1}$ Westaby recommends a sustained Valsalva manoeuvre to forcibly inflate the lung against the chest wall with breathing suspended until the purse string suture is tied. ${ }^{8}$ He goes on to suggest that auscultation of breath sounds after drain removal yields the same information as a check chest radiograph, and redrainage is unnecessary for "small" residual pneumothoraces. Donovan, in the discussion after the paper by Etoch et $a l^{3}{ }^{3}$ recommends drain removal in expiration with a second operator pinching closed the patient's nares and covering the mouth in order to prevent inspiration as the drain is removed. The authors rejected this approach on the grounds of strenuous objection from patient advocates! As three of our complications were recurrent pneumothoraces after drain removal, this is an area that merits further study to clarify the optimal approach to drain removal.
Another area of potential complication is dislodgement of the drain after insertion. This occurred in two of our patients and was also described by Collop et al ${ }^{7}$ and Etoch et al. ${ }^{3}$ The ATLS manual does not emphasise the importance of meticulously securing the drain in place with a combination of sutres and adherent dressings. ${ }^{1}$ This is emphasised by Westaby in his A User's Guide to Thoracic Drainage, ${ }^{8}$ and is a point well worth making.

This study discovered two drains that were replaced after kinking and clotting, thus becoming non-functional. The policy in our department with regard to drain size is in line with ATLS recommendations-that is, a \#36-40 French (F) drain. Westaby recommends a \#26 F or larger drain for haemothoraces and a \#20 F or larger for simple pneumothoraces. ${ }^{8}$ Collop et al described a $36 \%$ complication rate with small bore drain placements (\#14 F or less) compared with a $9 \%$ rate for standard tube thoracostomy placements. ${ }^{7}$ The use of a large bore (\#36 F or greater) drain is likely to reduce the complications associated with the drain becoming kinked or clotted off.

It is common practice to employ low pressure, high volume, suction for pneumothoraces that fail to respond to drainage alone. Complications can be encountered if a low volume, high pressure pump (Roberts type) is used. These include inability to clear a large volume air leak, ${ }^{8}$ and localised aspiration of lung into the drain resulting in pulmonary infarction. ${ }^{20}$ These complications can be simply avoided by using a low pressure, high volume pump.

\section{Conclusion}

The rationale for using tube thoracostomy in the treatment of many chest injuries, including simple pneumothorax and haemothorax, is well established. In particular ATLS recommends that all traumatic pneumothoraces be treated by tube thoracostomy on the basis that any simple pneumothorax left untreated could convert into a life threatening tension pneumothorax. ${ }^{1}$ ATLS also recommends that acute haemothorax, sufficiently large to appear on chest radiography, is best treated with a large calibre chest drain. The drain evacuates blood, reduces the risk of a clotted haemothorax, and provides a means of continuously monitoring blood loss.

Recently, Johnson has challenged the necessity of tube thoracostomy for small or moderate sized traumatic pneumothoraces on the basis of the high morbidity associated with the procedure. ${ }^{2}$ The most serious complications encountered are those associated with incorrect drain insertion, in particular the use of a closed technique and a trocar. Insertion of the drain using an open technique as described in the ATLS manual should eliminate these complications; none were encountered in this study.

The next important group of complications are infective, particularly empyema thoracis. Tube thoracostomy is a surgical procedure and as such full aseptic technique should be employed with appropriate wound care on the 
ward. The role of prophylactic antibiotics in reducing the incidence of empyema is still unclear. A prospective trial of antibiotic prophylaxis versus placebo would be useful to assess this question. This study encountered one case of empyema (representing a rate of $2 \% /$ tube thoracostomy) which did not result in long term morbidity.

The final group of complications analysed was the "positional" group. As stated previously meticulous care in drain anchorage and possibly a different technique of drain removal could have reduced these. There is a difference in opinion in the literature regarding which technique is best at preventing pneumothoraces after drain removal; this needs further investigation. None of this group of complications resulted in significant medium to long term morbidity, although the patients did have to endure a repeated procedure that elongated their length of admission.

In summary, although there are areas needing further study in an attempt to reduce the complication rate of tube thoracostomy, this study reveals no persuasive evidence to support a selective reduction in the indications for the procedure which could lead to an increased risk of tension pneumothoraces developing on the ward. A larger study to confirm or refute these findings needs to be undertaken before any change in established, safe practice.

Conflict of interest: none. Funding: none.

1 Committee on Trauma, American College of Surgeons. Advanced trauma life support. Chicago: American College of Surgeons, 1997.
2 Johnson G. Traumatic pneumothorax: is a chest drain always necessary? F Accid Emerg Med 1996;13:173-4.

3 Etoch SW, Bar-Natan MF, Miller FB, et al. Tube thoracostomy - factors related to complications. Arch Surg 1995;130:521-6.

4 Helling TS, Gyles NR, Eisenstein CL, et al. Complications following blunt and penetrating in 216 victims of chest trauma requiring tube thoracostomy. F Trauma 1989;29: trauma requ

5 Eddy AC, Luna GK, Copass M. Empyema thoracis in patients undergoing emergent closed tube thoracostomy for thoracic trauma. Am $\mathcal{F}$ Surg 1989;157:494-7.

6 Chan L, Reilly K, Henderson C, et al. Complication rates of tube thoracostomy. Am f Emerg Med 1997;15:368-70.

7 Collop NA, Kim SK, Sahn SA. Analysis of tube thoracostomy performed by pulmonologists at a teaching hospital. Chest 1997;112:709-13.

8 Westaby S. A user's guide to thoracic drainage. Gosport: Sherwood Medical, 1992

9 Hyde J, Sykes T, Graham T. Reducing morbidity from chest drains. BMF 1997;314:914-15.

10 Millikan JS, Moore EE, Steiner E, et al. Complications of tube thoracostomy for acute trauma. Am $\mathcal{F}$ Surg 1980;140: $738-41$.

11 Hewett FC. Thoracocentesis: the plan of continuous aspiration. $B M Y 1876 ; \mathbf{i}: 317$.

12 Shapira OM, Aldea GS, Kupferschmidj J, et al. Delayed perforation of the esophagus by a closed thoracostomy tube. Chest 1993;104:1897-8.

13 Fraser R. Lung perforation complicating tube thoracostomy: pathological description of 3 cases. Hum Pathol 1988;19:518-23.

14 Singh JK, Newman MA. Pulmonary artery catheterisation - an unusual complication of chest drain insertion. Aust N Z F Surg 1994;64:513-14.

15 Gerard PS, Kaldawi E, Litani V, et al. Right sided pneumothorax as a result of a left sided chest tube. Chest 1993;103: $1602-3$

16 Quak JM, Szatmaria A, Van Den Anker JN. Cardiac tamponade in a preterm neonate secondary to a chest tube. Acta Paediatrica 1993;82:490-1.

17 Nichols RL, Smith JW, Musik AC, et al. Preventative antibiotic usage in traumatic thoracic injuries requiring closed tube thoracostomy. Chest 1994;106:1493-8.

18 Grover FL, Richardson JD, Fewel JG, et al. Prophylactic antibiotics in the treatment of penetrating chest wounds. $\mathcal{F}$ Thorac Cardiovasc Surg 1977;74:528-36.

19 Fallon WF Jr. Post traumatic empyema. I Am Coll Surg 1994;179:483-92.

20 Stanley TL, Tench WD. Lung entrapment and infarction by chest drain suction. Radiology 1977;122:307. 